\title{
Design and Performance of a Dual-Polarity Instrument for Ion Soft landing
}

Pei $\mathrm{Su}^{1}$, Hang $\mathrm{Hu}^{1}$, Jonas Warneke ${ }^{1,4}$, Mikhail E. Belov ${ }^{2}$, Gordon A. Anderson ${ }^{3}$, Julia Laskin $^{1}$

1. Department of Chemistry, Purdue University, 560 Oval Drive, West Lafayette, Indiana 47907, United States

2. Spectroglyph, LLC, Kennewick, Washington 99338, United States

3.GAA Custom Engineering, LLC, Benton City, Washington 99320, United States

4. Wilhelm-Ostwald-Institut für Physikalische und Theoretische Chemie, Universität Leipzig, Linnestr. 2, 04103 Leipzig, Germany

Corresponding author: Julia Laskin, Tel: 765-494-5464, Email: jlaskin@purdue.edu

Table of Contents:

\section{Experimental Section}

Figure S1. Time-dependence of the ion currents of mass-selected $\mathrm{Ru}(\mathrm{bpy})_{3}{ }^{2+}$ and $\mathrm{WPOM}^{3-}$ in $\mathrm{ESI} \times 2$ or $\mathrm{ESI}(+/-)$ modes recorded over one hour time period. Stable currents are typically obtained over many hours of ion deposition.

Figure S2. Ion kinetic energy distributions (KEDs) of $\mathrm{Ru}(\mathrm{bpy}) 3_{3}{ }^{2+}$ and $\mathrm{WPOM}^{3-}$ acquired at different voltages applied to the conductance limit $\left(\mathrm{V}_{\mathrm{cl}}\right)$.

Figure S3. Visible spectra of Neutral Red (NR) in acidic and basic solutions.

Figure S4. Visible spectra of dropcasted acidic and basic NR methanol solutions.

Figure S5. Extraction ESI-MS spectra of the layer prepared by co-deposition of $\mathrm{Ru}(\mathrm{bpy})_{3}{ }^{2+} / \mathrm{MoPOM}^{3-}$. 


\section{Experimental Section}

\section{Chemicals}

Tris(2,2'-bipyridyl)dichlororuthenium(II) hexahydrate $\left(\mathrm{Ru}(\mathrm{bpy})_{3} \cdot 6 \mathrm{H}_{2} \mathrm{O}\right.$, CAS: 50525-27-4), Neutral Red $\left(\mathrm{C}_{15} \mathrm{H}_{17} \mathrm{ClN}_{4}\right.$, CAS: 553-24-2), sodium phosphotungstate tribasic hydrate $\left(\mathrm{Na}_{3}\left[\mathrm{PW}_{12} \mathrm{O}_{40}\right] \cdot x \mathrm{H}_{2} \mathrm{O}, \mathrm{CAS}\right.$ : 12026-98-1), sodium phosphomolybdate hydrate $\quad\left(\mathrm{Na}_{3}\left[\mathrm{PMo}_{12} \mathrm{O}_{40}\right] \cdot x \mathrm{H}_{2} \mathrm{O}, \quad \mathrm{CAS}: \quad 1313-30-0\right)$ and $1 \mathrm{H}, 1 \mathrm{H}, 2 \mathrm{H}, 2 \mathrm{H}-$ perfluorodecanethiol (FSAM, $\mathrm{CF}_{3}\left(\mathrm{CF}_{2}\right)_{7} \mathrm{CH}_{2} \mathrm{CH}_{2} \mathrm{SH}, \mathrm{CAS}$ : 34143-74-3) were purchased from Sigma-Aldrich (St. Louis, MO) and were used to demonstrate the instrument performance.

\section{FSAM Surface Preparation}

An FSAM on a gold surface was prepared as follows: a $10 \times 10 \mathrm{~mm}$ gold coated silicon wafer $(100 \mathrm{~nm}$ Au layer and $5 \mathrm{~nm}$ Ti adhesion layer, Platypus Technologies, Madison, WI) was ultrasonically washed in methanol, cleaned by a UV Ozone cleaner and immersed in a glass scintillation vial containing a $15 \mathrm{mM}$ solution of FSAM in methanol. The monolayer was allowed to assemble for at least $12 \mathrm{~h}$ and then ultrasonically washed for $5 \mathrm{~min}$ in methanol. The surface was subsequently rinsed with methanol, dried under nitrogen and mounted on the sample holder in the soft landing chamber.

\section{Surface Characterization}

Infrared reflection absorption spectroscopy (IRRAS) measurements were performed using a Bruker Tensor II FTIR spectrometer (Bruker Optics, Billerica, MA) equipped with a liquid nitrogen cooled mercury-cadmium-telluride (MCT) detector. Surfaces were mounted onto an $80^{\circ}$ reflection unit in the chamber of the spectrometer. IRRAS spectra were acquired at a spectral resolution of $4 \mathrm{~cm}^{-1}$ using p-polarized light. Each spectrum was collected by averaging 2000 scans corresponding to a 5-minute acquisition time. The experimental IRRAS plots were processed using water subtraction and baseline-correction options of the OPUS software.

Visible spectroscopy characterization of on the surfaces was performed using 
a R600-8-VisNIR reflectance probe connected to a BLK-CXR spectrometer and a SL1 Tungsten-Halogen visible-NIR light source (Stellarnet, Tampa, FL). Visible spectrum of a clean Au-FSAM surface was used as the reference. The spectra were collected using the SpectraWiz software.

Visible spectroscopy characterization of solutions was performed using an QP450-1-XSR optical fiber patch cords connected to USB2000+UV-VIS spectrometer and a DH-2000-DUV-TTL Deuterium Halogen light source (Ocean Optics, Largo, FL). Visible spectrum of methanol was used as the reference. The spectra were collected using the OceanView software.

Extraction ESI-MS analysis of the surface was performed by dissolving the deposited material with $80 \mu \mathrm{L}$ methanol and analyzed using Q-Exactive HF-X Hybrid Quadrupole-Orbitrap Mass Spectrometer (Thermo Fisher Scientific GmbH, Bremen, Germany). Sample was introduced into the MS inlet by direct infusion from a capillary $(50 \mu \mathrm{m} \mathrm{ID}, 150 \mu \mathrm{m}$ OD) at a flow rate of $\sim 0.5 \mu \mathrm{L} / \mathrm{min}$. Typical mass spectrometer conditions on Q-Exactive were as follows: electrospray voltage: $+/-3 \mathrm{kV}$, capillary temperature: $250{ }^{\circ} \mathrm{C}$, RF funnel level: 100 . Mass spectra were acquired over 150-2000 $\mathrm{m} / \mathrm{z}$ at 240000 resolution $(\mathrm{m} / \mathrm{z} 400)$. 


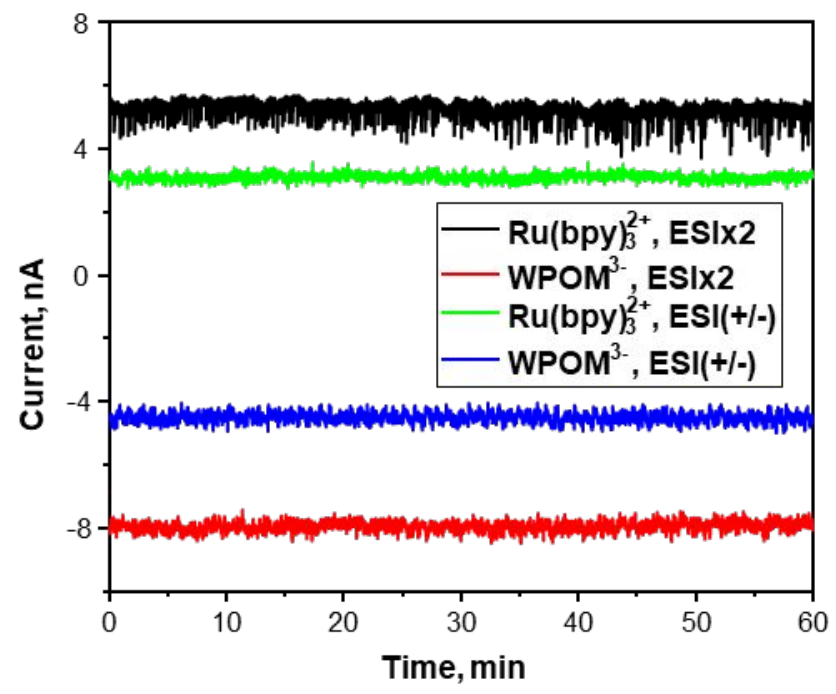

Figure S1. Ion current stabilities of mass-selected $\mathrm{Ru}(\mathrm{bpy})_{3}{ }^{2+}$ and $\mathrm{WPOM}^{3-}$ in $\mathrm{ESI} \times 2$ or ESI(+/-) modes over one hour time period. 

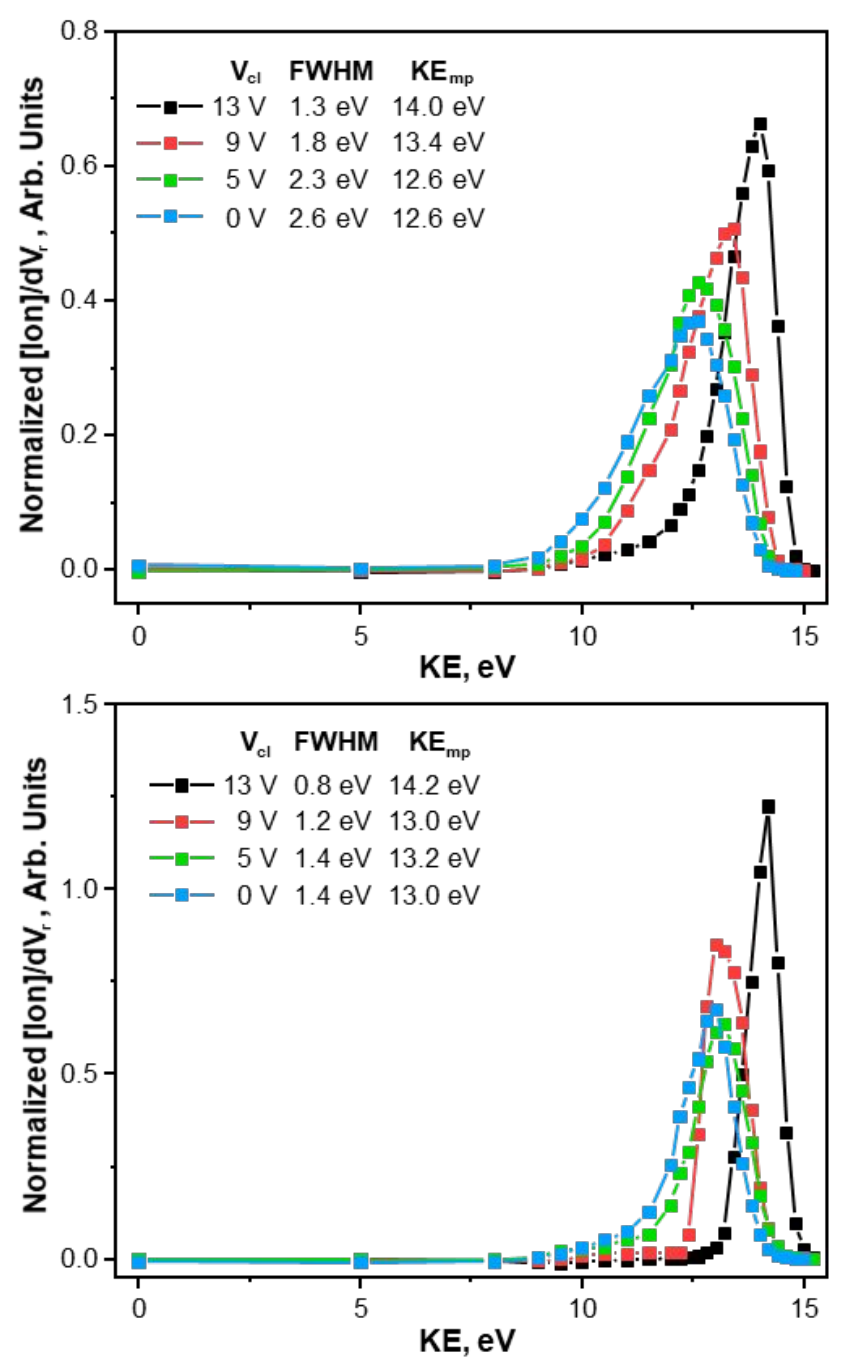

Figure S2. Ion kinetic energy distributions (KEDs) of Ru(bpy) $3^{2+}$ (a) and $\mathrm{WPOM}^{3-}$ (b) acquired at different voltages on the conductance limit $\left(\mathrm{V}_{\mathrm{cl}}\right)$. The flatapole bias was kept at $+14 \mathrm{~V} /-14 \mathrm{~V}$ for $\mathrm{Ru}(\mathrm{bpy})_{3}{ }^{2+}$ and $\mathrm{WPOM}^{3-}$, respectively. The FWHM and most probable kinetic energy $\left(\mathrm{KE}_{\mathrm{mp}}\right)$ of each $\mathrm{KED}$ plot was shown on the top left corner of each figure. 


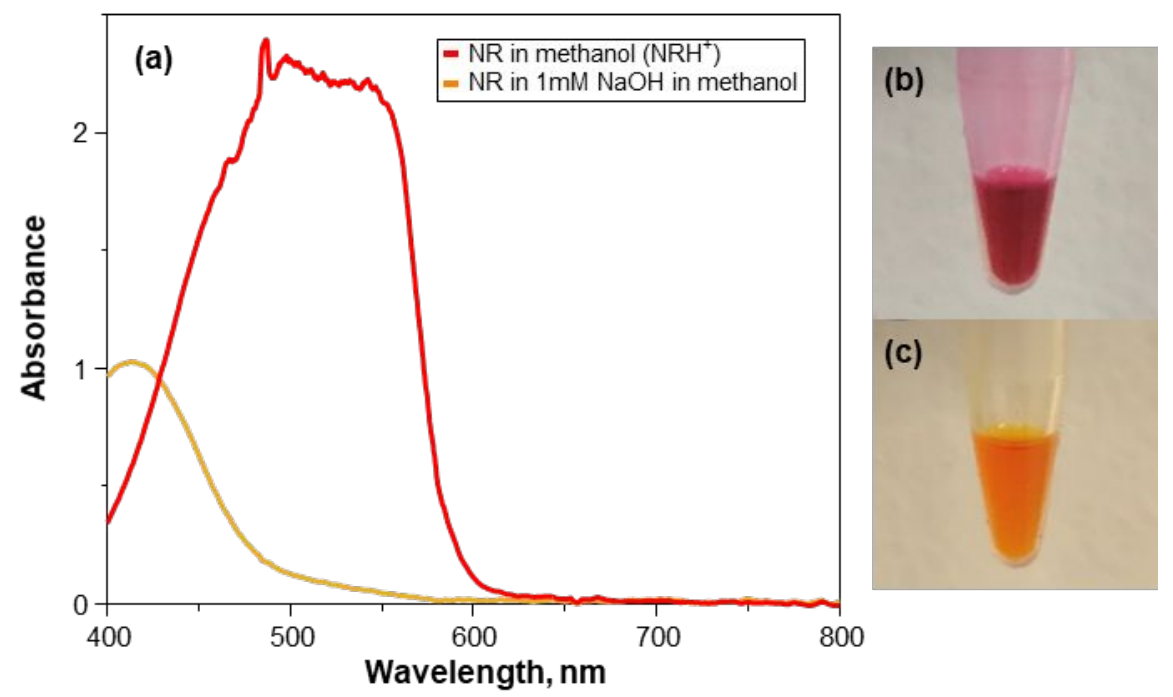

Figure S3. (a) Visible spectra of $1 \mathrm{mM}$ Neutral Red in methanol (red) and in a solution of $10 \mathrm{mM} \mathrm{NaOH}$ in methanol (yellow). Optical images of the solutions are shown on the right: (b) NR in methanol, (c) NR in $10 \mathrm{mM} \mathrm{NaOH}$ in methanol. 


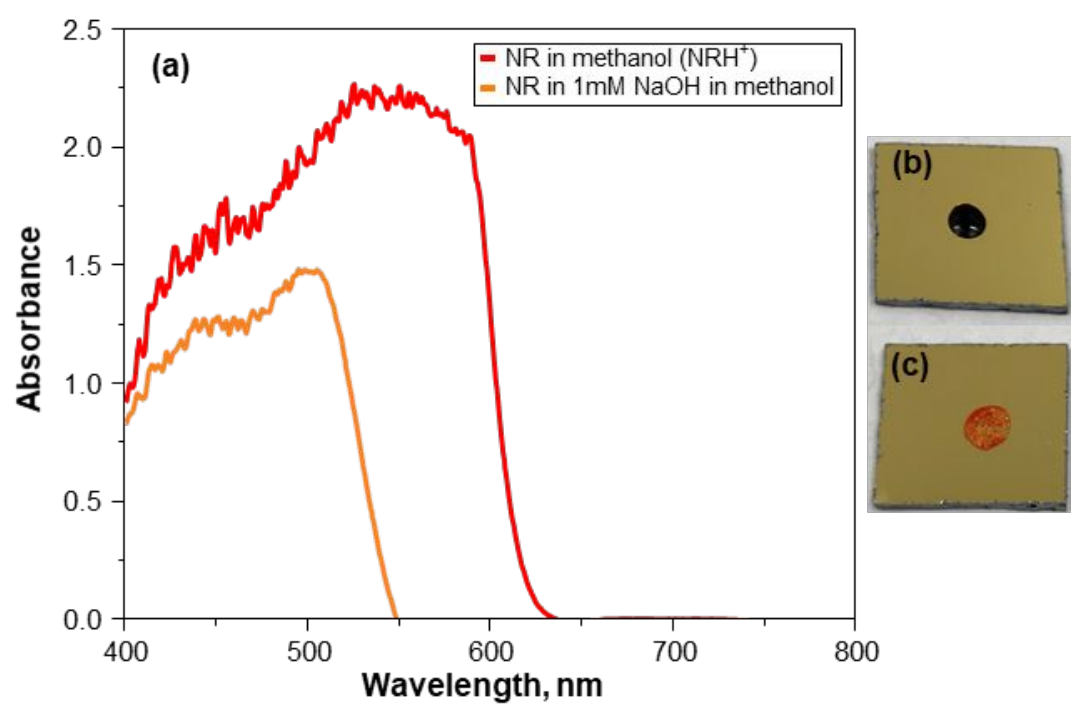

Figure S4. (a) Visible spectra of solutions of $1 \mathrm{mM}$ Neutral Red in methanol (red) and in a solution of $10 \mathrm{mM} \mathrm{NaOH}$ in methanol (yellow) dropcasted onto FSAM surfaces. Optical images of the dropcasted spots are shown on the right: (b) NR in methanol, (c) NR in $10 \mathrm{mM} \mathrm{NaOH}$ in methanol. 
S-8
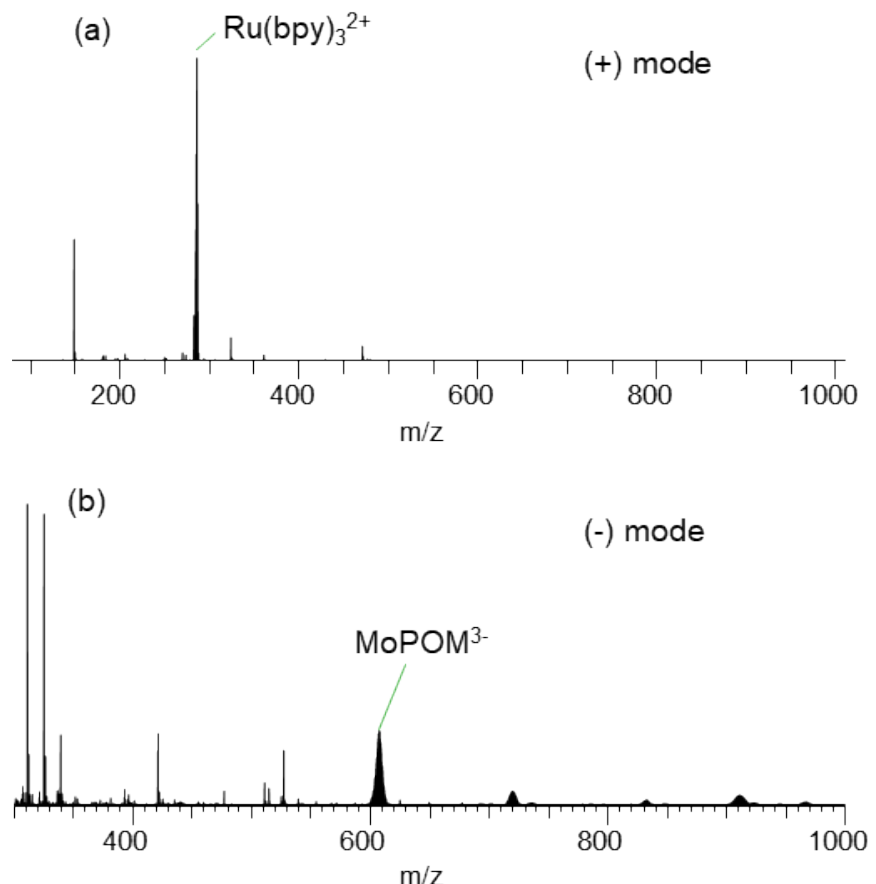

Figure S5. Extraction ESI-MS spectra of the layer prepared by co-deposition of $\mathrm{Ru}(\mathrm{bpy})_{3}{ }^{2+} / \mathrm{MoPOM}^{3-}$ in positive (a) and negative (b) modes. 